Antônio Carlos Gomes do Espírito Santo ${ }^{1}$

Oswaldo Yoshimi Tanaka ${ }^{1}$

${ }^{1}$ Centro de Ciências da Saúde, Departamento de Medicina Social,

Universidade Federal de Pernambuco. Av. Prof.

Morais Rego $s / n^{\circ}$, prédio do CCS/UFPE, Cidade

Universitária. 50670-420

Recife PE.

ag.santo@yahoo.com.br

\title{
Financiamento, gasto e oferta de serviços de saúde em grandes centros urbanos do estado de São Paulo (Brasil)
}

\author{
Health care financing, expenditure and supply \\ in great urban centers in the state of São Paulo (Brazil)
}

\begin{abstract}
Challenges to implantation of SUS doutrinary and operative principles in great urban centers, have stimulated the developing of strategies focused on cities over 100.000 inhabitants and had raised researches on this setting. This article approaches health financing and expenditure evolution in São Paulo cities over 500.000 inhabitants, excluded the state capital, from 2000 to 2006. These data were compared with health services production. The main data sources were Seade Foundation, Siops and Datasus database. Conclusions pointed out to an increase in health expenditure higher than global income and expense increasing. The low PSF coverage and the increasing of middle and high complexity procedures production found in this study might reflect the tradictional medical-assistance model reproduction. Indicators dissimilarity presented by different cities bring up the necessity of case studies, as simple as multiple ones, in order to better understand and to identify the determinants of these differences.
\end{abstract}

Key words Health financing and expenditure, Health integrality, Health care in great urban centers
Resumo Os desafios que se apresentam para implantação dos princípios doutrinários e operativos do SUS nos grandes centros urbanos têm motivado o desenvolvimento de estratégias particularmente voltadas para os municípios de porte populacional acima de 100.000 habitantes e suscitado o incremento de pesquisas que os elege como objeto de estudo. Neste artigo, buscou-se apreender a evolução do financiamento e do gasto com saúde nos municípios paulistas com população superior a 500.000 habitantes, excluído o município da capital, entre 2000 e 2006, estabelecendo um paralelo com a oferta de serviços ao longo deste periodo, utilizando-se para tanto os dados da Fundação Seade, do Siops e do Datasus. Concluise que há um gasto crescente com saúde superior ao crescimento da receita e da despesa global. Considerando os baixos valores de cobertura do PSF e o crescimento experimentado pela produção de procedimentos de média e alta complexidades, os recursos parecem direcionados para reprodução do modelo médico-assistencial tradicional. A heterogeneidade dos indicadores apresentados pelos diversos municípios apontam para a necessidade de estudos de casos, tanto simples quanto múltiplos, com vistas à identificação dos determinantes dessas diferenças.

Palavras-chave Financiamento e gasto em saúde, Integralidade em saúde, Atenção à saúde em municipios de grande porte 


\section{Introdução}

A reforma sanitária empreendida no Brasil a partir dos anos 70 deu-se num contexto de grave crise econômica e política e da eclosão dos movimentos pela redemocratização do país, sendo finalmente implementada no começo dos anos 90 , já dentro de um quadro de forte pressão dos organismos internacionais, particularmente dos integrantes do Consenso de Washington, para que os países em desenvolvimento adotassem o receituário neoliberal ${ }^{1,2}$. Tais considerações se mostram necessárias quando o propósito é compreender os desdobramentos dessa reforma, independentemente do foco e do ângulo de interesse do estudo, uma vez que reside nesta contextualização a explicação para as aparentes e não poucas contradições que emergem das análises.

As questões do financiamento e do gasto, bem como dos modelos de atenção, vêm fazendo parte das agendas de pesquisa sobre o Sistema Único de Saúde (SUS) talvez com a mesma frequência daquelas temáticas voltadas à avaliação dos seus princípios doutrinários e operativos. As mudanças ocorridas ao longo destes quase vinte anos de implantação do Sistema, decorrentes de injunções políticas e econômicas, da necessidade de ajustes nos mecanismos de gestão pública da saúde e do próprio acúmulo nesta área de conhecimento, produziram sensíveis alterações nas ênfases, na direção do olhar investigativo e no detalhamento imprimido à exploração daqueles objetos.

Desse modo, nos primeiros anos da década de 90, a par da preocupação com a extensão da cobertura e da descentralização, os estudos sobre financiamento e gasto se concentraram no acompanhamento dos volumes totais de recursos repassados pela União para os estados e municípios e para a resposta a este aumento no aporte financeiro, em termos da oferta de procedimentos disponibilizados. A demonstração das iniquidades geradas por um cálculo que privilegiava a produção de serviços era a tônica das pesquisas deste período, bem como da insuficiência e da instabilidade da base financeira do sistema ${ }^{3}$.

Com o intuito de fazer frente a essa problemática, medidas de considerável impacto foram implementadas nos anos de transição para o século atual. Uma delas diz respeito à alteração da fórmula de cálculo para estabelecimento do valor repassado pela União aos municípios, instituída a partir da Norma Operacional Básica de 1996 (NOB-96) e que passou efetivamente a vigorar em 1998, com instituição de um Piso de Atenção Básica, regido por uma lógica distribu- tiva de natureza demográfica e epidemiológica. A outra ocorreu em 2000, quando foi votada a Emenda Constitucional no 29 (EC 29), que assegurou um piso mínimo de investimento em saúde por parte das três esferas de governo. Estas mudanças suscitaram a realização de vários estudos avaliativos que se dedicaram a conferir o real cumprimento dos dispositivos baixados, bem como a avaliar o seu impacto na redução das desigualdades entre estados e regiões, contribuindo para tanto o destaque que o tema da equidade passou a assumir em escala mundial e particularmente nos países do Terceiro Mundo ${ }^{4,5}$.

Tendo em vista a flagrante heterogeneidade das condições de vida e de atenção à saúde presentes nos diferentes recantos do território nacional $^{6,7}$, a necessidade de particularizar os achados dessas pesquisas se mostra de fundamental importância, sob pena de não se conseguir apreender essa diversidade de situações e portanto não se obter o conhecimento necessário para intervir adequadamente nos problemas e acompanhar o impacto das ações propostas.

Considerando o elevado nível de urbanização verificado nas últimas décadas e as características especiais que assume a problemática de saúde nos grandes centros urbanos, conglomerados populacionais em que estratégias basilares da política nacional de saúde, a exemplo do Programa de Saúde da Família, se veem frontalmente desafiadas, o estudo do financiamento e do gasto em municípios de grande porte demográfico tem mobilizado pesquisadores de várias regiões do país ${ }^{8}$. O estado de São Paulo, unidade mais populosa da federação, detentor do maior produto interno bruto (PIB) do país e polo nacional de atração para a alta tecnologia de atenção à saúde, tem se constituído em um set privilegiado para a realização desses estudos. Nas conclusões da pesquisa realizada no estado de São Paulo como parte dos estudos de linha de base do Programa de Expansão e Consolidação do Programa de Saúde da Família (Proesf) ${ }^{9}$, são pontuadas as diferenças encontradas até mesmo entre os municípios integrantes de um mesmo cluster, constituído pela similitude de características, entre elas o porte populacional, o que instiga o desdobramento dessas categorias em conglomerados mais homogêneos e uma estratégia que promova uma análise comparativa entre seus próprios componentes.

O objetivo deste artigo, portanto, é o de descrever o padrão de financiamento e de gasto em saúde em municípios paulistas com população superior a 500.000 habitantes, excluído o municí- 
pio da capital, em face de suas características especialíssimas, as quais tornariam inviáveis as comparações pretendidas, bem como o modelo de atenção à saúde sustentado financeiramente por esses recursos. Nesse sentido, busca-se identificar a evolução da despesa pública com saúde, a participação nesta despesa dos diversos entes federativos e a produção das diferentes categorias de procedimentos ao longo dessa série histórica.

\section{Métodos}

Trata-se de uma pesquisa quantitativa, de natureza descritiva, retrospectiva e longitudinal, na qual se verifica a evolução das variáveis selecionadas no período 2000 a 2006 em cada um dos oito municípios de São Paulo com população superior a 500.000 habitantes, excluído o município da capital. Quatro deles localizam-se na Região Metropolitana de São Paulo: Guarulhos, Osasco, Santo André e São Bernardo. Os demais são Campinas, Ribeirão Preto e Sorocaba, sedes de macrorregiões de saúde, e São José dos Campos, localizada na região do Vale do Paraíba.

As variáveis selecionadas foram: receita municipal por habitante (RMH); despesa pública com saúde por habitante (DPSH); proporção dos recursos municipais no pagamento da despesa com saúde; percentual do orçamento municipal aplicado em saúde; taxa de cobertura do Programa de Saúde da Família; percentuais dos procedimentos de Atenção Básica, especializados e de alta complexidade na produção ambulatorial; média de internações por cem habitantes.

Uma das fontes de dados utilizadas foi o Sistema de Informações sobre Orçamentos Públicos em Saúde (Siops); no respectivo endereço eletrônico (http://portal.saude.gov.br/portal/saude/ profissional/area.cfm?id_area=1671) fizemos uso do endereço "Indicadores", trabalhando com os dados relativos à despesa empenhada a partir de 2000. A opção por esta categoria de despesa deveu-se, em parte, ao fato de ser aquela disponível ao longo de todo o período coberto pelo estudo. Além disto, representa o total das despesas feitas no respectivo ano, por cuja quitação o poder público assume responsabilidade, ao contrário da categoria despesas liquidadas, as quais não incluem os restos a pagar. Também foram utilizadas as informações recolhidas e sistematizadas pelo Departamento de Informação e Informática do Sistema Único de Saúde (Datasus) nos bancos de dados do Instituto Brasileiro de Geografia e Estatística (IBGE), Sistema de Infor- mações Ambulatoriais (SIA-SUS), Sistema de Informações Hospitalares (SIH-SUS) e Sistema de Informações da Atenção Básica (Siab). O ano de 2000 foi selecionado como marco inicial do período de estudo em face das importantes mudanças ocorridas no financiamento do Sistema Único de Saúde no final dos anos 90, já referidas na Introdução e ainda pela coincidência com o início de funcionamento do Siops e das mudanças na contabilidade de procedimentos implantadas pelo Datasus. O ano de 2006 foi escolhido para finalizar a série por ser o último ano com informações totalmente disponibilizadas quando da coleta de dados da pesquisa. $\mathrm{Na}$ análise foram considerados os valores absolutos e relativos das frequências, bem como sua média, mediana e amplitude de variação.

\section{Resultados e discussão}

\section{Evolução da despesa com saúde por habitante}

Os municípios participantes do estudo experimentaram, entre 2000 e 2006, uma taxa de crescimento da DPSH entre 46,83\% e 89,14\% (Gráfico 1), o que significa dizer que todos cresceram acima da inflação acumulada no período, que foi de 37,81\%, segundo o Índice Nacional de Preços ao Consumidor (INPC).

O aumento dos gastos públicos per capita com saúde no Brasil não se constitui fenômeno recente, uma vez que estudos baseados em séries históricas que cobriram a segunda metade dos anos 1990 já demonstravam esta tendência. Este é o caso do crescimento deste gasto por parte dos municípios do estado de Mato Grosso, na ordem de 3,5 vezes maior do que o crescimento da receita e da despesa total, identificado no estudo de Scatena e Tanaka ${ }^{4}$. No entanto, ao contrário do aumento da receita ocorrido nos municípios de médio e pequeno portes, desde a promulgação da Constituição de 1988, aqueles com população acima de 200.000 habitantes registraram crescimento da receita abaixo da média nacional. Em contrapartida, a despesa municipal com saúde experimentou um incremento diretamente proporcional ao porte populacional, chegando a uma taxa de $90 \%$ nos municípios entre 500.000 e um milhão de habitantes ${ }^{10}$.

Como se verifica no Gráfico 1, os percentuais de crescimento da DPSH nos municípios em estudo mostraram-se, em geral, mais elevados naqueles que possuíam valores abaixo de $\mathrm{R} \$ 150,00$, 


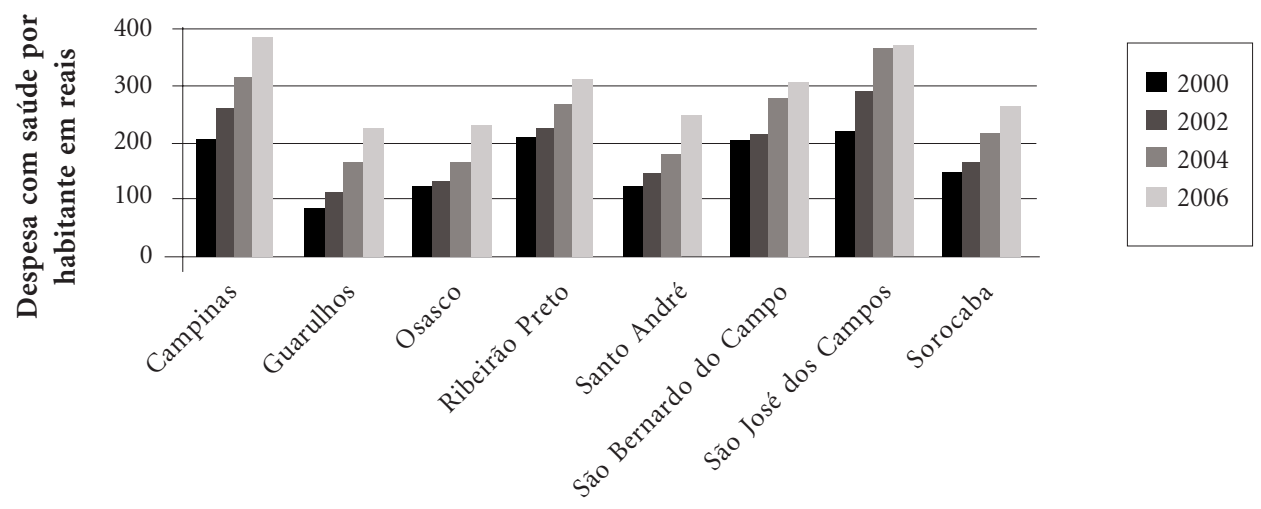

Municípios

Gráfico 1. Evolução da despesa pública com saúde por habitante em municípios paulistas selecionados em 2000, 2002, 2004 e 2006.

Fonte: Siops.

em 2000, situação de Guarulhos, Osasco, Santo André e Sorocaba. Tais municípios, a despeito do elevado crescimento relativo, chegaram a 2006 ainda entre aqueles com valores relativamente mais baixos de DPSH.

Em contrapartida, municípios como Ribeirão Preto, São José dos Campos e São Bernardo, que possuíam no ano 2000 valores de DPSH acima de $\mathrm{R} \$ 200,00$, apesar de um crescimento relativo menos acentuado, chegaram ao final do período entre os municípios com valores mais elevados de DPSH.

Campinas destoa de ambos os padrões, uma vez que já possuía em 2000 um dos valores mais altos daquele indicador e apresentou, além disso, um crescimento ao longo do período na ordem de $85 \%$, o que o fez chegar a 2006 na condição de município do grupo com valor mais elevado de DPSH.

A análise da DPSH em 2006 mostra um intervalo de variação que vai de $\mathrm{R} \$ 204,08$ em Osasco a R \$377,74 em Campinas, revelando a desigualdade das situações encontradas. Dentro desse intervalo situavam-se, em 2006, 60\% do total de municípios paulistas, estando 20\% deles acima destes valores de DPSH e 20\% abaixo. Osasco e Guarulhos, com valores abaixo de R\$230,00, aparecem como as menores DPSH, enquanto São José dos Campos e Campinas lideram o grupo, com valores acima de $\mathrm{R} \$ 370,00$.

O maior valor de DPSH encontrado - R\$ 377,74 em Campinas - ainda se encontra abaixo do gasto público per capita do Brasil em saúde, estimado por Carvalho ${ }^{11}$, para 2006, em R $\$ 422,49$.
Quanto aos dados disponibilizados pelo Siops sobre as despesas nacionais, regionais e dos estados, os mais recentes referem-se a 2004. De acordo com esta informação, o gasto público per capita com saúde do Brasil, naquele ano, foi de R\$ 358,54 . O Sudeste alcançou $\mathrm{R} \$ 360,57$, constituindo-se no maior valor regional de DPSH entre as regiões do país. Nesta região, o estado de São Paulo ocupou o segundo lugar, com R\$383,48, logo abaixo do Rio de Janeiro, que teve um gasto de $\mathrm{R} \$$ 439,96 . O maior valor, em âmbito nacional, foi alcançado pelo estado do Acre, com R \$495,77, e o menor foi o do Pará, com R\$211,60.

Tanto os valores estimados por Carvalho ${ }^{11}$ quanto aqueles fornecidos pelo Siops fornecem uma ideia de grandeza, sendo, portanto, de alguma ajuda no processo de análise. No entanto, não se prestam a uma comparação direta em face das restrições decorrentes da diferença cronológica dos dados e da heterogeneidade de situações que eles incorporam, ao incluir municípios com características muito distintas. Além disso, as despesas contidas no seu cálculo incluem os gastos da União e dos estados para manutenção do pessoal e das unidades de saúde ainda não municipalizadas. Desta forma, abrangem outras despesas com saúde além daquelas sob responsabilidade do município, indicador utilizado neste estudo e em cujo cálculo são levadas em consideração somente as transferências intergovernamentais para a saúde e os recursos resultantes da arrecadação de impostos e taxas.

A participação dos recursos municipais entre 2000 e 2006 no pagamento das despesas com saúde 
mostra uma redução em três dos municípios estudados - Sorocaba, Campinas e Guarulhos -, indicando um aumento do percentual de transferências intergovernamentais no pagamento da despesa no período estudado (Tabela 1). Nos demais municípios, identifica-se tendência inversa, com uma magnitude de aumento que em alguns deles, como São Bernardo e Ribeirão Preto, é mínima, e em outros, como Osasco e São José dos Campos, mostra-se considerável. As mudanças no mecanismo de repasse do Ministério para os municípios pode ter influenciado esta situação, mas não há indícios de que a tendência tenha obedecido a critérios relacionados com o percentual verificado em 2000 ou, como se verá adiante, com o porte do orçamento.

Tomando por base o ano de 2006, verifica-se que os percentuais de participação de recursos municipais na despesa pública com saúde variaram de 54,19\% em Sorocaba a 80,29\% em Guarulhos, com valor percentual médio de $68,19 \%$ e mediana de $66,28 \%$.

Todos os municípios do grupo de estudo participaram com recursos próprios em mais de 50\% da despesa total com saúde sob responsabilidade do município, sendo que em três deles esta participação superou os 70\% (Tabela 1).

Confrontando os dados da Tabela 1 com aqueles do Gráfico 1, verifica-se que em municípios como São José dos Campos esse alto percentual de recursos municipais aplicados é responsável em 2006 por uma DPSH que se apresenta como uma das mais elevadas do grupo. Já Guarulhos, mesmo participando com 80,29\% do pagamento da despesa, detém ainda uma DPSH que se encontra entre as mais baixas. Campinas, por sua vez, deteve a maior DPSH mesmo com uma proporção dos recursos municipais na despesa pouco acima de $60 \%$.

De acordo com um estudo do Departamento de Economia da Saúde, do Ministério da Saúde, que utilizou dados do Siops, citado em documento do Conselho Nacional de Secretários de Saúde ${ }^{12}$, a tendência geral nos últimos anos é de crescimento da participação dos estados na despesa com saúde, passando de $18,5 \%$, em 2000 , para 25,6\%, em 2004; e dos municípios, de 21,7\% para 24,5\%. A participação do Ministério da Saúde, em contrapartida, teria caído no mesmo período de 59,8\% para 49,9\%. Estes valores não se prestam a uma comparação direta com o indicador que está sendo utilizado pelos mesmos motivos especificados anteriormente com referência aos dados nacionais e estaduais referentes ao gasto per capita.

\section{Evolução do percentual do orçamento municipal aplicado em saúde}

Em se tratando dos municípios que foram objeto desta pesquisa, à exceção de Guarulhos, com 14,60\%, e Ribeirão Preto, com 14,46\%, todos os municípios paulistas já tinham um percentual do orçamento municipal aplicado em saúde, em 2000, que os colocava bem acima do percentual mínimo estabelecido pela Emenda Constitucional 29, que para aquele ano exigia um gasto mínimo de recursos próprios dos municípios

Tabela 1. Participação do município no custeio da despesa total com saúde, receita municipal por habitante e percentual do orçamento municipal aplicado em saúde em municípios paulistas selecionados - 2000-2006.

\begin{tabular}{|c|c|c|c|c|c|c|}
\hline \multirow{2}{*}{ Municípios } & \multicolumn{2}{|c|}{$\begin{array}{c}\text { Participação do município no } \\
\text { custeio da despesa total com saúde }\end{array}$} & \multirow{2}{*}{$\begin{array}{l}\text { Receita municipal } \\
\text { por habitante em } \\
2006\end{array}$} & \multicolumn{3}{|c|}{$\begin{array}{l}\text { Orçamento municipal } \\
\text { aplicado em saúde }\end{array}$} \\
\hline & $\begin{array}{c}\text { Percentual de } \\
\text { participação } 2006\end{array}$ & $\begin{array}{l}\text { Crescimento } \\
2000-2006\end{array}$ & & 2000 & 2006 & $\begin{array}{c}\text { Crescimento } \\
2000-2006\end{array}$ \\
\hline Guarulhos & 80,29 & $-16,43$ & 735,22 & 14,60 & 19,8 & 5,20 \\
\hline Osasco & 68,36 & 11,88 & 678,47 & 27,49 & 27,1 & $-0,39$ \\
\hline Ribeirão Preto & 64,21 & 2,27 & 805,21 & 14,46 & 26,0 & 11,54 \\
\hline Santo André & 63,10 & 7,48 & 870,32 & 17,85 & 19,9 & 2,05 \\
\hline São Bernardo & 79,96 & 1,78 & $1.232,80$ & 22,37 & 20,7 & $-1,67$ \\
\hline
\end{tabular}

Fonte: Siops. 
igual a 7\%, evoluindo este percentual progressivamente até chegar a 15\% em 2004. Em quatro municípios o percentual aplicado naquele ano ultrapassou 20\% (Tabela 1). Em três deles - Campinas, São Bernardo e São José dos Campos, além de Osasco, que aplicava em 2000 um percentual de $18,74 \%$ - houve uma queda discreta ao longo do período, com o maior percentual de decréscimo ocorrendo em Campinas (- 2,14\%). Estes valores de crescimento negativo, contudo, não chegaram a produzir, em 2006, nenhum percentual de aplicação de recursos próprios abaixo de $17 \%$. Dos quatro municípios do grupo que apresentaram incremento neste percentual, destacase Ribeirão Preto, que cresceu 11,54 pontos percentuais no período. Assim sendo, o intervalo de variação observado em 2006 é de 16,9\%, em Sorocaba, a 27,3\% em São José dos Campos, com média de 21,98\% e mediana de 20,3\%.

Segundo Bremaeker ${ }^{10}$, já em 1998 o percentual do orçamento municipal com saúde aplicado pelos municípios brasileiros com população entre 500 mil e um milhão de habitantes era de $18,9 \%$. Particularmente no caso dos municípios paulistas, $97 \%$ já cumpriam a Emenda Constitucional 29 em 2003, de acordo com o estudo publicado pelo Centro de Estudos de Cultura Contemporânea ${ }^{9}$.

\section{O papel desempenhado pelo porte do orçamento municipal no valor dos indicadores estudados}

Apesar dos problemas existentes na elaboração e na execução dos orçamentos municipais e que terminam por limitar o seu uso como ferramenta privilegiada de gestão, o seu porte, principalmente depois da aprovação da Emenda Constitucional 29, com seu efeito vinculante, representa um referencial básico para a avaliação da capacidade do gasto em saúde ${ }^{13}$.

A diferença entre os municípios que integram o estudo, no tocante aos valores da receita municipal, em valores tanto absolutos como por habitante, é bastante acentuada, verificando-se que a maioria dos municípios acha-se entre os valores de $\mathrm{R} \$ 600,00$ e $\mathrm{R} \$ 900,00$ de $\mathrm{RMH}$, estando os demais situados acima de $\mathrm{R} \$ 1.000,00$ (Tabela 1).

Por motivos óbvios, o grau de influência do percentual de recursos próprios aplicado em saúde sobre a DPSH se acha na razão direta do porte do orçamento municipal. Assim é que entre São José dos Campos e Osasco, que têm os maiores percentuais de recursos próprios aplicados em saúde, o primeiro apresenta o valor mais ele- vado de DPSH, enquanto o segundo detém o valor mais baixo. Esta dedução é reforçada pela situação de Campinas, que, mesmo tendo o terceiro menor percentual de aplicação de recursos próprios, se encontra entre os valores mais altos de DPSH, graças a um orçamento que é o maior entre os municípios considerados, ultrapassando R\$ 1 bilhão. Situação inversa é a de Guarulhos, que detém a segunda menor RMH do grupo e participa no custeio da despesa com o maior percentual $(80,29 \%)$, como pode ser visto na Tabela 1.

\section{Cobertura \\ do Programa de Saúde da Família (PSF)}

Todos os municípios iniciam a série com valores de cobertura do PSF abaixo de 10\% (Gráfico 2), alguns com o percentual zerado, como é o caso de Ribeirão Preto e São José dos Campos. Osasco e Sorocaba chegaram a 2006 sem ultrapassar aquele patamar. Campinas, Guarulhos e São Bernardo, por sua vez, experimentaram, mediante grandes saltos ocorridos, para cada um deles, em diferentes anos da série, um rápido crescimento, chegando a 2006 com percentuais acima de $30 \%$. São José dos Campos, que nos primeiros anos da série, em 2002, esteve entre municípios do grupo que detinham os percentuais mais altos, permaneceu estacionário, chegando em 2006 com 16\%. Do mesmo modo, Ribeirão teve um aumento de apenas quatro pontos percentuais, passando de $29 \%$, em 2002, para 33\%, em 2006. São Bernardo, que tinha o percentual mais elevado em 2002 (37,5\%), experimentou uma queda gradual, chegando a 2006 em torno de $32 \%$. No conjunto, os percentuais máximos, em 2006, situavam-se abaixo de 35\%.

O Informe da Atenção Básica de setembro/ outubro de 2003 mostra que a cobertura média dos 27 municípios brasileiros com população entre 500.000 e dois milhões de habitantes era de $22 \%$ e que a meta estipulada para eles era de no mínimo 40\%, situando, portanto, com eloquência a condição dos municípios integrantes desta pesquisa no cenário nacional (Programa de Expansão e Consolidação do Programa de Saúde da Família $\left.{ }^{14}\right)$.

Mesmo levando em consideração o tratamento diferenciado dado aos municípios com população acima de 100.000 habitantes no que diz respeito às taxas de cobertura, haja vista que instrumentos como a Portaria 396/GM de $2003^{15}$ fixe em 50\% a cobertura do Programa de Saúde da Família para a concessão da maior faixa de 


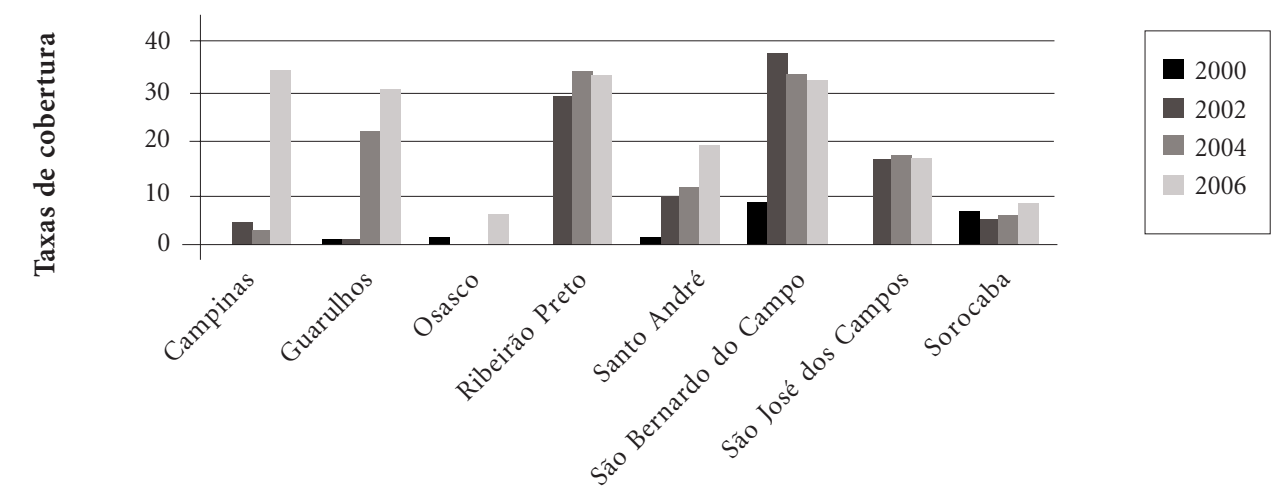

Municípios

Gráfico 2. Taxa de cobertura do Programa de Saúde da Família em municípios paulistas selecionados nos anos de 2000, 2002, 2004 e 2006.

Fonte: Datasus.

incentivo, verifica-se que tais municípios ainda se acham bem aquém daquele valor. Deve ser lembrado, ainda, que o caráter substitutivo das estratégias tradicionais de Atenção Básica pelo Programa de Saúde da Família continua sendo uma diretriz perseguida e reforçada pelo Sistema Único de Saúde, como se depreende da leitura que se faz da portaria 648/GM de $2006^{16}$, a qual aprova a Política Nacional de Atenção Básica.

A realidade apontada pelo indicador de cobertura representa, evidentemente, o grau de implantação da estratégia PSF nos municípios e não, necessariamente, o seu nível de investimento na Atenção Básica nem a efetividade dessa estratégia, haja vista que indicadores como cobertura vacinal e de pré-natal mantiveram-se, em todos os municípios paulistas estudados, superiores a 90\% ao longo dos anos da série. No entanto, demonstra em que medida está se operando a conversão do modelo assistencial, propósito maior de iniciativas como a do Proesf ${ }^{14}$, com vigência estipulada para o período 2002-2009.

Baixas coberturas de PSF têm sido encaradas como um traço comum aos municípios de grande porte populacional, devido ao custo para implantação das equipes, à resistência para superação de uma cultura médico-assistencial arraigada e à dificuldade de captar profissionais capacitados, principalmente médicos, como destacam Favoreto et al. ${ }^{7}$. Vale lembrar, no entanto, que a cobertura média de PSF em 2006, no estado de São Paulo como um todo, era de 30,9\%.

\section{Produção de procedimentos ambulatoriais por níveis de complexidade}

Os percentuais relativos ao volume dos procedimentos ambulatoriais de Atenção Básica gerados nos anos extremos da série estudada (2000 e 2006), em relação ao total de procedimentos ambulatoriais naqueles anos, variaram em 2000 entre 31,27\%, em Campinas, a 69,36\%, em São Bernardo, mas em seis dos municípios esse percentual ultrapassava 50\% (Tabela 2). A média desse percentual, que em 2000 é de 52,46\%, com mediana de 51,53, alcança valores próximos da média do estado, de $51,92 \%$, estando já bem abaixo da média nacional, que era de $62,51 \%$.

Em 2006, esta situação altera-se substancialmente, com os limites inferior e superior da amplitude de variação caindo para 15,8\% e 56,8\%, respectivamente. Apenas um município - São Bernardo - manteve o percentual acima de $50 \%$, sofrendo, mesmo assim, uma queda de mais de 12 pontos percentuais.

Em todos os municípios ocorre uma queda nesses percentuais, sendo que as mais acentuadas referem-se a Sorocaba e a São José dos Campos, com valores acima de 30 pontos percentuais. Nos demais, com exceção de Guarulhos, essas quedas foram sempre superiores a dez pontos percentuais. A média desce para 32,21\%, correspondendo a uma queda de 19,71 pontos percentuais. Nesse período, o percentual do estado caiu para 38 e o do Brasil para $49,60 \%$, equivalendo, 


\begin{tabular}{|c|c|c|c|c|c|c|c|}
\hline \multirow{13}{*}{ 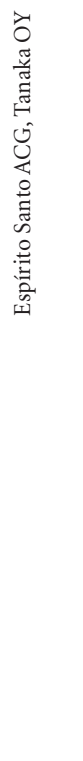 } & $\begin{array}{l}\text { Tabela 2. Distribuição } \\
\text { em municípios paulis }\end{array}$ & $\begin{array}{l}\text { entual d } \\
\text { lecionad }\end{array}$ & $\begin{array}{l}\text { procedil } \\
\text { no esta }\end{array}$ & $\begin{array}{l}\text { ntos ambulatori } \\
\text { de São Paulo e }\end{array}$ & $\begin{array}{l}\text { segundo os ní } \\
\text { Brasil - } 2000\end{array}$ & $\begin{array}{l}\text { de comple } \\
066 .\end{array}$ & dade \\
\hline & \multirow[b]{2}{*}{ Municípios } & \multicolumn{2}{|c|}{ Atenção Básica } & \multicolumn{2}{|c|}{ Procedimentos especializados } & \multicolumn{2}{|c|}{ Alta complexidade } \\
\hline & & 2000 & 2006 & 2000 & 2006 & 2000 & 2006 \\
\hline & Campinas & 31,27 & 15,8 & 47,05 & 34,4 & 21,68 & 49,8 \\
\hline & Guarulhos & 50,40 & 45,7 & 36,38 & 49,1 & 13,22 & 5,2 \\
\hline & Osasco & 66,30 & 44,1 & 24,98 & 28,1 & 8,72 & 27,8 \\
\hline & Ribeirão Preto & 51,27 & 24,3 & 30,57 & 29,8 & 18,16 & 45,9 \\
\hline & Santo André & 40,42 & 28,8 & 48,07 & 23,6 & 11,51 & 47,6 \\
\hline & São Bernardo & 69,36 & 56,8 & 30,18 & 42,3 & 0,46 & 0,9 \\
\hline & São José dos Campos & 59,94 & 26,0 & 33,86 & 43,2 & 6,20 & 30,7 \\
\hline & Sorocaba & 50,79 & 16,2 & 39,83 & 30,2 & 9,38 & 53,6 \\
\hline & Estado de São Paulo & 51,92 & 38,0 & 39,67 & 36,5 & 17,41 & 25,5 \\
\hline & Brasil & 62,51 & 49,6 & 31,92 & 33,5 & 5,57 & 16,9 \\
\hline
\end{tabular}

Fonte: Datasus.

respectivamente, a uma queda de 14 e 13 pontos percentuais.

A média da alta complexidade era de $17,47 \%$, situando-se, portanto, ainda na média do estado, que era de $17,41 \%$, mas já bem acima da média nacional, que era de 5,57\%. Em 2006, essa média passa para $32,68 \%$, superando a média do estado e duplicando o valor nacional.

A escalada dos procedimentos de média e alta complexidades nos municípios de grande porte é um fenômeno verificado desde os anos 90. Scatena e Tanaka ${ }^{4}$ identificaram nos municípios de Mato Grosso com populações superiores a 50.000 habitantes, entre 1994 e 1998, um significativo aumento dos procedimentos ambulatoriais de média e alta complexidades, principalmente daqueles serviços de complementação diagnóstica e terapias especializadas. Ao mesmo tempo, detectaram uma interrupção no crescimento dos gastos com as ações de vigilância epidemiológica, imunização e atos não médicos (AVEIANM) e uma franca redução nos gastos com consultas médicas e odontológicas.

No que toca aos gastos federais, as estimativas de Vianna et al. ${ }^{17}$ eram de que, apesar do notável crescimento dos gastos com a alta complexidade no período entre 1995 e 2003, pelo menos até 2010 estes não ultrapassariam os gastos com Atenção Básica.

É preciso ter claro que esses números não significam uma redução em termos absolutos do volume de procedimentos de Atenção Básica produzido. Sendo valores relativos, indicam um aumento percentual dos volumes de procedimentos de média e de alta complexidades. Entre os motivos que têm sido relacionados para esse fenômeno encontra-se a demanda reprimida desses procedimentos. Essa repressão de demanda se dava, em parte, pela própria restrição de acesso. Na medida, portanto, em que tal acesso passa a ser facilitado, principalmente pela extensão da cobertura da Atenção Básica via PSF, essa demanda começa a ser satisfeita. A organização dos mecanismos de regulação e o repasse de recursos federais aos municípios para o atendimento dessas modalidades de atenção também vão responder pelo aumento na produção desses procedimentos. O modelo assistencial ainda prevalente na Atenção Básica, individual, curativista, baseado na demanda espontânea e no ato médico, é também citado como determinante desse fenômeno. É possível que a forma de remuneração dos procedimentos de média e alta complexidades, que ainda obedece à lógica da produção, ao contrário dos procedimentos de Atenção Básica, vinculados muito mais a uma lógica de repasse per capita populacional, exerça alguma pressão na contabilização dos procedimentos.

\section{Disponibilidade de internações}

Analisando-se as internações por local de internação (Tabela 3), observa-se uma variação em 2000 entre 3,3 e 10,7 internações por cem habitantes, com média de 6,42 e mediana de 5,85. Dos oito municípios, quatro situavam-se abaixo da média do estado, que era de 5,56. O parâmetro colocado previsto na Portaria 1.101 (Portaria GM $1.101^{18}$ ) situa-se entre sete e nove internações por cem habitantes. 
Tabela 3. Internações por cem habitantes segundo local de internação e local de residência em municípios paulistas selecionados - 2000 e 2006.

\begin{tabular}{|c|c|c|c|c|}
\hline \multirow[b]{2}{*}{ Municípios } & \multicolumn{2}{|c|}{$\begin{array}{c}\text { Internações - local de } \\
\text { internação/100 habitantes }\end{array}$} & \multicolumn{2}{|c|}{$\begin{array}{c}\text { Internações - local de } \\
\text { residência/100 habitantes }\end{array}$} \\
\hline & 2000 & 2006 & 2000 & 2006 \\
\hline Campinas & 6,8 & 6,7 & 6,5 & 4,7 \\
\hline Guarulhos & 7,9 & 3,7 & 5,5 & 4,3 \\
\hline Osasco & 4,9 & 2,9 & 5,0 & 3,5 \\
\hline Ribeirão Preto & 10,7 & 10,1 & 6,4 & 6,0 \\
\hline Santo André & 3,9 & 4,1 & 3,5 & 3,6 \\
\hline São Bernardo & 3,3 & 3,2 & 3,6 & 4,0 \\
\hline São José dos Campos & 4,9 & 4,4 & 4,9 & 4,3 \\
\hline Sorocaba & 9,0 & 8,9 & 5,5 & 5,2 \\
\hline
\end{tabular}

Fonte: Datasus.

Na maioria dos municípios, verifica-se uma estabilidade dos valores ao longo do período analisado, à exceção de Guarulhos e Osasco. Tal estabilidade deve-se, provavelmente, à regulação estabelecida pelo número de cotas de autorização de internação hospitalar (AIH) que estavam definidas no teto estadual. Assim, houve uma adequação do faturamento ao teto físico-financeiro definido pelo MS para o estado de São Paulo.

As diferenças acentuadas entre o número de internações por local de ocorrência e por residência em Campinas, Ribeirão Preto e Sorocaba devem se referir à demanda que recebem dos demais municípios situados nas regiões de saúde das quais são sedes.

\section{Conclusões}

Com a designação de "municípios de grande porte", independentemente do quantitativo populacional que se estabeleça como ponto de corte, abriga-se uma grande variedade de situações no que diz respeito a financiamento e gasto com saúde, bem como em relação ao perfil do modelo de atenção à saúde. Tais diferenças, pouco evidenciadas quando esses municípios são agrupados em conglomerados e submetidos a uma análise comparativa com aqueles de médio e de pequeno portes, mostram-se bastante explícitas num confronto intragrupo.

Nessas condições, a similitude do porte populacional não impacta de forma homogênea os indicadores selecionados, o que pode ser visto pela acentuada diferença entre os municípios com contingentes populacionais aproximados, como Campinas e Guarulhos ou como Ribeirão Preto e Sorocaba.

Fica claro, por conseguinte, que outras variáveis devem ser consideradas em estudos de corte analítico, na busca de explicação para os achados, entre elas as que se relacionam com a densidade tecnológica disponível, o porte do orçamento municipal, o estágio de desenvolvimento social e econômico do município e da região onde se acha inserido, além da qualidade da gestão em saúde posta em prática, haja vista a grande disparidade observada entre esses indicadores naqueles municípios.

Embora com níveis diversificados de esforços, os municípios em questão demonstram empenho no sentido de atenuar essas desigualdades, haja vista o percentual de recursos dos seus orçamentos que vêm aplicando na saúde, notadamente aqueles com uma defasagem histórica de despesa com saúde por habitante ao longo do período. Fica evidenciado, de qualquer modo, que embora se identifique um aumento real da despesa com saúde ao longo do período, esta ainda se acha aquém dos valores praticados em países com padrões elevados de saúde. Nesse sentido, Piola e Servo ${ }^{19}$ afirmam que os cálculos para o Brasil apontam um dispêndio total (público e privado) de cerca de 7,6\% do PIB com saúde. O "gasto" privado responde por $58,4 \%$ do total e o "gasto" direto das famílias atinge $64,1 \%$ da fatia privada. Ou seja, como parcela do PIB gastamos valores próximos aos dos países da Organização para Cooperação e Desenvolvimento Econômico (OCDE), como Inglaterra e Espanha, mas, ao contrário destes, aqui a 
participação das fontes públicas é muito menor (41,6\% do gasto total, segundo a OMS, para 2002). Além disso, como nosso produto interno bruto per capita é menor do que o desses países, percentuais equivalentes do PIB correspondem a gastos per capita em saúde consideravelmente menores.

A estabilidade dos diferenciais referentes ao número de internações por habitantes ao longo dos anos estudados, regulado que é pelas cotas de AIH, demonstra que certas iniquidades resistem aos espaços e fóruns de pactuação instituídos pelo Sistema.

No nível municipal, sendo já elevado o comprometimento orçamentário com a saúde, a expansão dessa base financeira estará na dependência do aumento de seus respectivos orçamentos, o que se acha na razão direta de uma ampliação da arrecadação de seus impostos ou de alterações na redistribuição fiscal entre os entes federados. Nesse momento, no entanto, as esperanças de um incremento dos valores da despesa com saúde parecem repousar no valor dos repasses do governo federal e na sua política de redistribuição desses recursos?.

No entanto, parece muito claro que nesses municípios a destinação do gasto não se acha pautada pela prioridade de investimento na Atenção Básica e, muito menos, pela Estratégia Saúde da Família, que se expandiu timidamente ao longo desses anos, de modo que os melhores resultados de cobertura alcançam pouco mais de $30 \%$. Pelo contrário: a média e principalmente a alta complexidade experimentaram uma expansão que produz uma inversão na distribuição proporcional dos tipos de procedimentos entre 2000 e 2006, consumindo a maior parte dos recursos disponíveis para a saúde.

Os dados parecem confirmar as conclusões de Scatena e Tanaka ${ }^{4}$ de que nos municípios de grande porte persiste o "modelo do Inamps", cen- trado na assistência médica curativa, individual, especializada e com alta densidade tecnológica. Por sua vez, Santos e Gerschman ${ }^{20}$ também constatam uma total dissociação, nos grandes centros urbanos, entre o modelo de saúde operante e aquele pretendido pelo SUS. Entendem que se caminha para um plano de cuidados básicos, preconizados pelo Banco Mundial, aliado a um estoque de maior complexidade cujo porte depende dos interesses e das pressões do mercado e da disponibilidade do setor público para adquirilos. Sendo este o perfil que se vislumbra numa etapa em que, para aquelas autoras, são os gestores que tomam as principais decisões, atuando de forma individual ou através dos seus órgãos de representação, nas diversas esferas de governo e nos diferentes colegiados de deliberação do sistema, deslocam para os trabalhadores, gerentes e usuários a esperança de produzir mudanças nesse quadro, mediante uma atuação articulada.

Cumpre-se, pelo visto, apenas em parte, prognósticos como aquele colhido em um estudo de abrangência nacional, realizado em 2000, empregando metodologia Delphi, no qual $60 \%$ dos participantes, representando diferentes categorias de atores do SUS, acreditavam que na primeira década do século XXI haveria sensível redução das desigualdades em saúde. Entre os que não partilhavam dessa perspectiva otimista, a maior proporção era de representantes do setor privado e da sociedade civil, integrantes da amostra. A maioria dos participantes do estudo previa forte desenvolvimento e incorporação tecnológica no setor, mas sem controle do poder público. Acreditavam eles que tal incorporação poderia reduzir o custo em saúde, mas admitiam que a possibilidade de universalização do acesso estaria condicionada a um elenco mínimo de ações, correspondendo quando muito àquelas assumidas pelo Sistema no final dos anos $90^{17}$.

\section{Colaboradores}

ACG Espírito Santo e OY Tanaka participaram igualmente de todas as etapas da elaboração do artigo. 


\section{Referências}

1. Silva PLB. Serviços de saúde: o dilema do SUS na nova década. São Paulo em Perspectiva 2003; 17(1):69-85.

2. Cordeiro H. Descentralização, universalidade e equidade nas reformas de saúde. Cien Saude Colet 2001; (6)2:319-328.

3. Novaes HMD. Avaliação de programas, serviços e tecnologias em saúde. Revista de Saúde Pública 2000; 3(5):547-559.

4. Scatena JHG, Tanaka OY. Utilização do Sistema de Informações Hospitalares (SIH-SUS) e do Sistema de Informações Ambulatoriais (SIA-SUS) na análise da descentralização da saúde em Mato Grosso. Informe Epidemiológico do SUS 2001; 10(1):19-30.

5. Souza RR. Redução das desigualdades regionais na alocação de recursos federais para a saúde. Cien Saude Colet 2003; 8(2):449-460.

6. Programa das Nações Unidas para o Desenvolvimento (PNUD), Instituto de Pesquisa Econômica Aplicada (Ipea). Relatório sobre o Desenvolvimento Humano no Brasil. Brasília: PNUD/Ipea; 1996.

7. Favoreto CAO, Daim S, Caetano R. Elementos tecnoassistenciais na análise da Atenção Básica à Saúde em grandes cidades no Brasil. Instituto de Medicina Social, Faculdade de Ciências Médicas, Universidade do Estado do Rio de Janeiro. [acessado 2007 nov 7]. Disponível em: http://www.sms.rio.rj.gov.br/ servidor/media/tecnoassistenciais2.doc

8. Brasil. Ministério da Saúde. Secretaria de Atenção à Saúde. Departamento de Atenção Básica. Projeto de Expansão e Consolidação do Programa de Saúde da Família. Brasília: MS/SAS/DAB; 2003.

9. Centro de Estudos de Cultura Contemporânea (Cedec). Consórcio Medicina USP: financiamento e gasto com saúde nos municípios paulistas com população superior a 100.000 habitantes (20002003). Cadernos de Atenção Básica: estudos descritivos. São Paulo: Cedec; 2006.

10. Bremaeker FEJ. Os gastos municipais com a saúde em 1998. Rio de Janeiro: Instituto Brasileiro de Administração Municipal (Ibam), Núcleo de Articulação Político-Institucional; 2000.

11. Carvalho G. Gasto com saúde no Brasil em 2006. [acessado 2007 out 22]. Disponível em: http://www. ensp.fiocruz.br/radis/55/pdf/gasto.co.saude.2006.pdf

12. Conselho Nacional de Secretários de Saúde (Conass). SUS: avanços e desafios. Brasília: Conass; 2006.

13. Wenceslau J. Pesquisa sobre rastreamento dos gastos públicos em saúde. Banco Mundial, 2005. [acessado 2007 nov 26]. Disponível em: www.fazenda. gov.br/spe/publicacoes/fiscal/seminarios/01 ApresentacaoSPEPETS.pdf
14. Programa de Expansão e Consolidação do Programa de Saúde da Família (Proesf). Avaliação Normativa da Estratégia de Saúde da Família no Brasil - 2001/2002. Informe da Atenção Básica 2003; 18. Brasília, 2003. [acessado 2007 nov 26]. Disponível em: dtr2004.saude.gov.br/dab/docs/publicacoes/ informes/psfinfo18.pdf.

15. Brasil. Ministério da Saúde. Portaria nº 396/GM, de 4 de abril de 2003. Reajusta os valores dos incentivos financeiros aos Programas de Saúde da Família, de Agentes Comunitários de Saúde e às Ações de Saúde Bucal no âmbito do Programa de Saúde da Família e dá outras providências. [acessado 2007 nov 26]. Disponível em: http://dtr2001.saude.gov. $\mathrm{br} / \mathrm{sas} /$ PORTARIAS/Port2003/GM/GM-396.htm

16. Brasil. Ministério da Saúde. Portaria nº 648/GM, de 28 de março de 2006. Aprova a Política Nacional de Atenção Básica, estabelecendo a revisão de diretrizes e normas para a organização da Atenção Básica para o Programa Saúde da Família (PSF) e o Programa Agentes Comunitários de Saúde (Pacs). [acessado 2007 nov 26]. Disponível em: http://dtr2004. saude.gov.br/dab/docs/legislacao/portaria_648_ 28_03_2006.pdf

17. Vianna SM, Nunes A, Góes G, Silva JR, Santos RJM. Atenção de alta complexidade no SUS: desigualdades no acesso e financiamento [relatório de pesquisa, v. I]. Brasília: MS/Ipea; 2005. (Projeto Economia da Saúde).

18. Brasil. Portaria GM $n^{\circ} 1.101$, de 12 de junho de 2002. Estabelece os parâmetros de cobertura assistencial no âmbito do SUS. Diário Oficial da União 2002; 13 jun. p. 36, seção 1 .

19. Piola S, Servo LM. Contas em saúde: um instrumento de apoio à gestão do SUS. Instituto de Pesquisa Econômica Aplicada (Ipea). Diretoria de Estudos Sociais. Brasília, 2005. [acessado 2007 out 8]. Disponível em: http://dtr2001.saude.gov.br/sctie/portal_ des/eventos/apresentacoes/contas_Luciana_Piola_ jornadas_2.ppt

20. Santos MAB, Gerschman S. As segmentações da oferta de serviços de saúde no Brasil: arranjos institucionais, credores, pagadores e provedores. Cien Saude Colet 2004; 9(3):795-806.

Artigo apresentado em 06/02/2008

Aprovado em 01/07/2008

Versão final apresentada em 16/07/2008 